\title{
Personality features and vulnerability to stress: a case study on hyperhidrosis
}

\author{
Agata Andò, ${ }^{1}$ Marzia Di Girolamo, ${ }^{1}$ Claudia Pignolo,${ }^{1}$ Alessandro Zennaro, ${ }^{1}$ Luciano Giromini, ${ }^{1}$ Amedeo Minichino, ${ }^{2}$ \\ Adriana Salatino, ${ }^{1}$ Rosalba Morese ${ }^{1,3}$
}

${ }^{1}$ Department of Psychology, University of Turin, Italy; ${ }^{2}$ Department of Psychiatry, University of Oxford, United Kingdom; ${ }^{3}$ Faculty of Communication Sciences, Università della Svizzera Italiana, Lugano, Switzerland

\begin{abstract}
By using the Rorschach test, self-reports, and psychophysiological measures, we investigated thoroughly the psychological functioning in a hyperhidrotic case. Erica, a young female with hyperhidrosis, was assessed in three times at one-week distance. First, specific tools assessing potential psychological and affective distress, and the Rorschach test were administered. About one week later, Electrodermal Activity was recorded during the exposure to a mild laboratory stress-inducing task. Finally, a magnetic resonance imaging

Correspondence: Marzia Di Girolamo, Department of Psychology, University of Turin, Via Po 14, 10123 Turin, Italy.

Tel.: +39.011.670.3060.

E-mail: marzia.digirolamo@unito.it

Citation: Andò, A., Di Girolamo, M., Pignolo, C., Zennaro, A., Giromini, L., Minichino, A., Salatino, A., \& Morese, R. (2019). Personality features and vulnerability to stress: a case study on hyperhidrosis. Research in Psychotherapy: Psychopathology, Process and Outcome, 22(1), 83-98. doi: 10.4081/ripppo.2019.344

Contributions: AA conceived and coordinated the study, interpreted the data and wrote the manuscript; MDG coordinated the study, inexam was performed in order to exclude medical conditions/neurological alterations for potential physiological anomalies. Erica tends to avoid living in the moment and prefers to experience close relationships in her inner world where she can rehearse the future and imagine different contexts and social situations without risks and embarrassment. She reports high capacities to perform goaldirected behaviors and clarity of emotions only in absence of stressful situations. The study has the merit to be the first to combine Rorschach data with physiological data in order to investigate the psychological functioning in a hyperhidrotic case.
\end{abstract} terpreted the data (in particular, the Rorschach data) and wrote the manuscript; $\mathrm{CP}$ analyzed data and wrote the manuscript; AZ, AS: contributed to the research design, wrote and reviewed the manuscript; LG helped interpreting Rorschach data and reviewed the manuscript; AM reviewed the manuscript; RM conceived and supervised the study.

Conflict of interest: the authors declare no potential conflict of interest.

Funding: none.

Acknowledgments: the authors would like to thank Dr. Maria Consuelo Valentini for the assistance and for providing them with access her lab and equipment.

Ethical statement: all procedures performed in studies involving human participants were in accordance with the ethical standards of the institutional and/or national research committee and with the 1964 Helsinki declaration and its later amendments or comparable ethical standards. The participant gave her written informed consent to participate in the study, which was approved by the Institutional Review Board of the University of Turin, Italy, and for the publication of the current case study.

Received for publication: 12 November 2018.

Revision received: 28 January 2019.

Accepted for publication: 1 February 2019.

This work is licensed under a Creative Commons Attribution NonCommercial 4.0 License (CC BY-NC 4.0).

CCopyright A. Andò et al., 2019

Licensee PAGEPress, Italy

Research in Psychotherapy:

Psychopathology, Process and Outcome 2019; 22:83-98

doi:10.4081/ripppo.2019.344
Key words: Hyperhidrosis; Electrodermal activity; Laboratoryinduced stress; Rorschach test; Psychological functioning.

\section{Introduction}

Hyperhidrosis is defined as excessive sweating outside the range required for normal thermal regulation (Vorkamp, Foo, Khan, Schmitto, \& Wilson, 2010). It usually occurs in childhood or adolescence and can affect primarily hands and soles of the feet. The estimated incidence of hyperhidrosis ranging from $0.6 \%$ to $2.8 \%$ of the population (with a higher representation of females than males, probably due to a greater awareness of women about their suffering Won et al., 1998 - leads to significant social and psychological distress - Cinà \& Clase, 1999; Ricchetti-Masterson et al., 2018). Hyperhidrosis can be idiopathic (primary) or secondary to systemic disease, metabolic disorder, febrile illness, neurological problems, use of certain drugs, cancer, and chronic infections (Mahendiran, Burkhart, \& Burkhart, 2009). Primary hyperhidrosis affects the eccrine sweat glands due to excessive sympathetic activity (Esen et al., 2005); although primary hyperhidrosis is not caused by another medical condition, it can be associated with other diseases such as Raynaud's syndrome, a vasomotor disease characterized by the change in skin color of the limbs due to the pallor, followed by cyanosis (Wigley \& Flavahan, 2016). When excessive sweating is localized (e.g., palms, 
soles, face, underarms, scalp), it is defined as focal hyperhidrosis, while excessive sweating involving the entire body is defined as generalized hyperhidrosis. The generalized hyperhidrosis is usually secondary to an underlying condition (most often an infectious, endocrine, or neurologic disorder) or may have a physiological basis with humidity, heat or exercise, leading to excessive sweating; differently, the focal hyperhidrosis is a idiopathic (primary) condition and may occur in healthy people (Mahendiran et al., 2009).

However, nowadays the pathophysiology of primary hyperhidrosis is not yet sufficiently understood (Haider \& Solish, 2005); although some physiologists claim it is caused by over-activity of the sympathetic nervous system (e.g., Vetrugno, Liguori, Cortelli, \& Montagna, 2003). Indeed, De Marinis and colleagues (2012) asserted that the primary hyperhidrosis was a complex dysfunction involving autonomic pathways beyond those associated to sweating. Other similar results discussed by Birner and coworkers (2000) reported elevated sympathetic skin responses to excitatory stimulation tests in hyperhidrotic subjects. Lin, Chee, Chen, and Cheng (1995) supposed that primary hyperhidrosis occurred due to a regulatory dysfunction. Manca, Valls-Sole, and Callejas (2000) described an enhancement of the sudomotor skin responses suggesting hyperexcitability of the somatosympathetic polysynaptic pathway involved in sweating. Iwase et al. (1997) suggested that an excessive response in sympathetic nerve activity to the palmar and plantar skin to both thermal and mental stimuli was responsible for the intense and abundant sweating.

Individuals with hyperhidrosis usually experience limitations in various functional domains as well as in the overall quality of life (e.g., Sayeed, Nyamekye, Ghauri, \& Poskitt, 1998); several problems were reported as associated to work limitations, disturbances in social life, and difficulties in relationship with spouse and recreational activities (Cina, Robertenson, Young, Cratier, \& Clae, 2006). Moreover, they reported low levels of motivation and effectiveness at work, moderate to severe depressive symptoms, and reduced confidence (Solish, Benohanian, \& Kowalski, 2005; Strutton, Kowalski, Glaser, \& Stang, 2004). Embarrassment, shame, insecurity, frustration, unhappiness, and sometimes depression are other feelings often reported by individuals with hyperhidrosis (e.g., Adar, Kurchin, Zweig, \& Mozes, 1977). Especially during adolescence, they may show difficulties in social and intimate relationships leading to reclusiveness and avoidance of social interactions and leisure activities. To cope with the excessive sweat soaking through their clothing, shoes, or handheld items, they use several strategies in a daily effort in order to hide their symptoms, such as using pads, towels, gloves, shields, absorbent tissues, layers of clothing, dark colors of clothing, bulky clothing, and/or frequent clothing changes (Glogau, 2001).

Overall, these previous studies (and their some contradictory findings) mentioned above focused mainly on the impact of hyperhidrosis on psychological well-being and its consequences, not investigating how people with hyper- hidrosis may react to the disease by using those cognitive and emotion regulation strategies related to personality features. While several studies were conducted to examine the physiologic basis of primary hyperhidrosis and related limitations in several functional domains as well as in the overall quality of life, only few studies investigated personality features in patients with this disease.

Although some authors concluded that the relationship between sweating pattern and emotional pattern, specific disorders, or family relationship history is absent (Karaca et al., 2007; Ruchinskas, 2002; Won et al., 1998), Ak (2013) found that individuals with hyperhidrosis were characterized by specific personality traits, temperament, and character features compared to controls, associated to lower purposefulness, resourcefulness, self-directedness and higher self-forgetfulness and self-transcendence. Moreover, Johnson and Mostaghimi (1995) reported the hyperhidrosis as the most frequent dermatological disorder in psychiatric subjects. Most importantly, we can note that some studies investigated the possibility of personality traits as an etiological factor for primary hyperhidrosis (Ak, 2013), while others either refuted etiological conclusions (Karaca et al., 2007; Won et al., 1998), or considered the psychological differences to be the result of hyperhidrosis and not its cause (Ruchinskas et al., 2002). The idea of this study arose from our thought that the impact of this illness on psychological well-being might depend on the personality functioning.

We focused on those cognitive and emotion regulation strategies that may be used for managing and coping this medical condition. Given that distinct methods engage and assess distinct psychological processes (Bornstein, 2015, 2017), we used a multimethod assessment including the Rorschach Performance Assessment System (R-PAS; Meyer, Erard, Erdberg, Mihura, \& Viglione, 2011), selfreports, and psychophysiological measures, in order to examine thoroughly the psychological functioning and the vulnerability to stress in a hyperhidrotic case. Our aim was to obtain information related to the conscious awareness and information not necessarily filtered through the self-schema. Specifically, we wondered how Erica perceived and described herself and what might be those irrational aspects influencing her behaviors. The vulnerability to stress is a complex construct including the interaction between cognitive, functions, implicit emotions, and physiological parameters; only integrating different tools it may be possible to investigate the multiple components associated to managing the hyperhidrosis.

Psychological functioning in hyperhidrosis was never investigated through the R-PAS, which is a performancebased test providing information about the whole person's personality functioning including also those implicit aspects influencing behaviors. Specifically, we investigated whether the stress and distress R-PAS variables were associated with increased sympathetic activity and provided information on psychological resources, in a case with hy- 
perhidrosis. In our opinion, the disabling impact of primary hyperhidrosis can not be isolated from the rest of the subject's resources and limitations.

\section{Materials and Methods}

Erica (fictitious name), an undergraduate student in Psychology, voluntarily accepted and was recruited at the University of Turin (Italy) in order to participate in an experimental study on the possible relationships between electrodermal activity (EDA) and Rorschach variables. When Erica had a first meeting i) to ensure inclusion and exclusion criteria (i.e., currently undertaking drugs, history of psychiatric disorders or neurological illness and psychological disorders, smoking, professional sport activities, and also a previous administration of the Rorschach test) and to obtain written consent (Erica gave the written informed consent to attend the original experimental study. Subsequently, she gave her approval to participate in the current experimental study, and to publish her single case study), ii) to check some psychophysiological data (e.g., skin conductance and heart rate) and potential physiological anomalies, experimenters observed an extremely elevated EDA signal and excessive sweating from Erica. In this context, Erica let the experimenters know that she was affected by hyperhidrosis.

After collecting these baseline psychophysiological data, examiners offered to Erica a follow-up meeting to discuss about her physical condition and they suggested her to attend a new experimental study to investigate the psychophysiological correlates of her illness. Examiners explained to Erica that such new experimental study would aim at examining specifically her medical condition; examiners described in details the approach of the study including several measures related to distinct operating domains: performances, and psychophysiological tests. Given that in the past years, Erica consulted several experts on skin disorders for understanding her hyperhidrosis' causes not obtaining a comprehensive explanation, she appeared to be enthusiastic to participate in the current experimental study aiming to examine thoroughly her psychophysical condition. Erica received a feedback on findings from examiners at the end of the study, after the data were obtained and interpreted.

One of the clinical psychologists who conducted the assessment met Erica in order to discuss about her medical disease. Erica spontaneously reported only some information on her life and her hyperhidrosis symptoms. Erica's hyperhidrosis was not caused by another underlying condition and/or as a side effect of a medication or procedure. Erica reported that hyperhidrosis occurred during the pre-pubertal period although she described hyperhidrosis as a difficult condition especially during adolescence. She consulted several experts on skin disorders in order to obtain an appropriate treatment; a doctor suggested her to undergo the sympathectomy that is treatment in which the sympathetic chain is interrupted; she refused this surgery. In describing her disease, she never reported previous requests of psychological support. A brief history of Erica's life is described below.

\section{Personal information, history and evolution of medical condition}

\section{How Erica describes her life with hyperhidrosis}

Erica is a 21-year-old female affected by the hyperhidrosis condition associated with Raynaud's phenomenon. Severe bilateral palmar and plantar sweating occur especially during social and stressful situation. Erica refers that when she is barefooted her hands and feet are dry, while the Raynaud phenomenon persists. The hyperhidrosis was for Erica an embarrassing condition that interfered with routine activities, caused emotional distress, and disturbed the overall social life. During her life, Erica has tried to manage her emotional inner world in order to be able to control those stressful situations that may cause the excessive sweating. Erica reports that going through life was hard given the impossibility to hide a sweaty handshake, soaking wet underarms, or dripping feet. Given that hyperhidrosis often caused socially isolation during adolescence, Erica decided to fight her discomfort and not to avoid social events and situation by starting to play the guitar in a symphony orchestra. She reports that the hyperhidrosis was not a problem while she was playing and that it was pleasant to use a string instrument. When Erica was 14 years old, she met her first boyfriend: she recognized this moment as a turning point of her life because, for her, staying in a relationship meant that someone had accepted her medical condition. Few months after being in this romantic relationship, she abandoned the orchestra ("I did not feel the need to play anymore").

During the last years, she consulted several experts on skin disorders in order to obtain an appropriate treatment, but they did not find a real solution to her problems related to the hyperhidrosis. However, Erica found a new, efficient way to deal with her discomfort, so that currently she is doing several sportive activities such as climbing, martial arts, and ski, in order to try to modulate her anxiety and stress associated to the excessive sweating ("Now I am a sportswoman").

Erica describes herself as a calm and shy person, with a tendency to withdrawal. She prefers to live in a fantastic inner world and not to show her feelings to other people. Moreover, Erica believes to have a unique and great memory ability leading her to remember vividly her previous emotional experiences ("My memory is related to my emotional word").

\section{Family relationship history}

\section{How Erica describes her family}

Erica is an only child and lives with her parents. She has a hostile relationship with her mother described as a person who tends to make others feel guilty; indeed, 
Erica's mother seems to be a very challenging adversary, because she often feeds upon the altruistic responses related to those preoccupations elicited in others. Erica's mother is described as attention-seeking, emotional overreacting, and suggestible, tending to over-dramatize situations and to impair family's relationships ("If my mother does not obtain attentions she could become crazy").

Erica reports some episodes during her childhood in which both parents caused her distress. Her mother tried to open her hands aggressively in order to wash them while Erica used to keep her fists strongly clenched. She remembers how her mother's attitude caused feeling of discomforts, fear, and irritation. Furthermore, she reports that her hands were dry when she was an infant but something changed over time. Her father, on the other hand, used to become upset when she wore socks to sleep or to stay at home, and demanded her to remove them ("He was often upset for wearing my socks, I do not know why. He is that kind of person that if he does not like something, neither the others have to do it").

Erica says how her parents frequently had irritable mood and behaviors whose causes and real motivations she did not understand. During childhood and adolescence, Erica participated in family arguments. Erica felt to be the focal point of the family because she was often involved in resolving the conflicts between her mother and father. Erica reports she experienced a painful episode while her parents were arguing against each other and her father shouted: "It is enough! I'm leaving!". While Erica was crying, her mother said, "You mustn't cry! You have to stop him". Erica did not react to her mother's request. Erica felt that she would have had a great responsibility in resolving such hostile situation. A few weeks later, Erica heard her uncle talking with her father: "If you leave out, it will be a hopeless pain for your daughter". Arguments between her parents decreased over time and her father never left his family. Overall, Erica experienced a high hardy atmosphere due to marital conflicts. She fretted that her parents would get divorced or that one of them would abandon her ("Often my family made me anxious. I had not to talk with my parents about their marital conflicts; my parents pretended that everything was fine").

\section{Procedure}

After the follow-up meeting, Erica was administered brief tools assessing potential psychological and/or affective distress and emotion dysregulation and the Rorschach task according to R-PAS guidelines (Time 1; T1). About one week later EDA was recorded during exposure to a mild laboratory stress-inducing task, involving a threephase baseline-stress-recovery trial laboratory induced stress (Time 2; T2). One week later after EDA recording, magnetic resonance imaging (MRI) exam was performed (Time 3; T3) in order to exclude medical conditions/neurological alterations for potential physiological anomalies.

\section{Measures}

As mentioned in the introduction, the approach used to study the case integrated several psychological tools, related to distinct operating domains: performances and psychophysiological tests. The incremental value of this approach has been pointed out from several authors over the years (e.g., see Ando' et al., 2017; Ando' et al., 2015; Bornstein, 2017; Campbell \& Fiske, 1959; Giromini et al., 2016a, 2016b; Meyer, 2018).

\section{Symptoms Checklist-90-R}

The Symptoms Checklist-90-R (SCL-90-R; Derogatis, 1994) is a self-report checklist developed to measure the psychological and/or affective distress. Each of the 90 items is scored on a 5-point Likert scale ranging from 0 (not at all) to 4 (extremely). The test comprises nine clinical scales (Somatization [SOM], Obsessive-Compulsive [O-C], Interpersonal Sensitivity [I-S], Depression [DEP], Anxiety [ANX], Hostility [HOS], Phobic Anxiety [PHOB], Paranoid Ideation [PAR], and Psychoticism [PSY]) and three indices (Global Severity Index [GSI], Positive Symptom Distress Index [PSDI], and Positive Symptom Total [PST]). The scores are expressed in T scores, so that scores higher than $65 \mathrm{~T}$ indicate the presence of psychological or affective distress. The studies of the psychometric properties of the SCL-90-R have provided satisfactory results with respect to both test-retest and internal reliability with alpha coefficients ranging from .79 to .90 for the different dimension scales in clinical and non-clinical sample (Horowitz, Rosenberg, Baer, Ureño, \& Villaseñor, 1998).

\section{The State-Trait Anxiety Inventory}

The State-Trait Anxiety Inventory (STAI-Y; Spielberger, Gorsuch, Lushene, Vagg, \& Jacobs, 1983) consists of 40 questions on a self-report basis and assesses two types of anxiety: state anxiety (Y1-FORM) and trait anxiety (Y2-FORM). The two forms of anxiety are separated in the inventory, and both consist of 20 separate questions. Each item is rated on a 4-point Likert scale ranging from 1 ("not at all") to 4 ("very much so"). Raw scores are transformed into $\mathrm{T}$ scores, which have a mean of 50 and standard deviation of 10 , so that $\mathrm{T}$ scores higher than 65 indicate the presence of state or trait anxiety. Internal consistency alpha coefficients were high ranging from .86 for high school students to .95 for military people (Spielberger, Gorsuch, Lushene, Vagg, \& Jacobs, 1983). For our study, we used STAI-Y1 to assess potentially nervousness or discomfort before starting the assessment.

\section{Toronto Alexithymia Scale}

The Toronto Alexithymia Scale (TAS-20; Bagby, Parker, \& Taylor, 1994) was developed to measure the alexithymia construct and it comprises three scales: i) Difficulty Identifying Feelings, ii) Difficulty Describing Feelings, and iii) Externally Oriented Thinking. Accord- 
ing to the author, raw scores at 51 or lower suggest the absence of alexithymia features, raw score at 61 or higher indicate alexithymic cases, whereas raw scores between 52 and 60 indicate borderline cases (Taylor, Bagby, \& Parker, 1997). TAS demonstrates a good internal consistency (Cronbach's alpha=.81; Bagby et al., 1994).

\section{Difficulties in Emotion regulation scale}

The Difficulties in Emotion Regulation Scale (DERS; Gratz \& Roemer, 2004; Giromini, Velotti, de Campora, Bonalume, \& Zavattini, 2012) is a 36 -item, self-report questionnaire comprising six subscales developed to detect multiple aspects of emotion dysregulation: i) Non-acceptance of emotional responses (Nonacceptance), ii) Difficulties engaging in goal directed behavior (Goals), iii) Impulse control difficulties (Impulse), iv) Lack of emotional awareness (Awareness), v) Limited access to emotion regulation strategies (Strategies), vi) Lack of emotional clarity (Clarity). Recently, Giromini, Ales, de Campora, Zennaro, and Pignolo (2017) provided equations to calculate age and gender adjusted T-scores, so that clinicians would easily interpret the resultant T-transformed, DERS scores, which have a mean of 50 and standard deviation of 10 . According to the authors, DERS scores between $65 \mathrm{~T}$ and $70 \mathrm{~T}$ indicate the presence of problems in emotion regulation, whereas scores higher than $70 \mathrm{~T}$ indicate significant problems in emotion regulation. DERS reported high alpha coefficients ranging from .80 to .89 for the different scales (Gratz \& Roemer, 2004).

\section{Questionnaire of Cognitive and Affective Empathy}

The Questionnaire of Cognitive and Affective Empathy (QCAE; Reniers, Corcoran, Drake, Shryane, \& Völlm, 2011) is a 31-item questionnaire comprising two scales, both with subscales, developed to assess both cognitive and affective components of empathy on a 4-point Likert scale. The Cognitive Empathy scale consists of two subscales: i) Perspective Taking (i.e., capability to put oneself in another person's shoes), and ii) Online Simulation (i.e., attempts to put oneself in another person's position by thinking the feeling of such person). Three subscales constitute the Affective Empathy scale: i) Emotion Contagion (i.e., automatic mirroring of other's feelings), ii) Proximal Responsivity (i.e., emotional responsive-ness to the feelings of other people who are close within the affective subject's context) and iii) Peripheral Responsivity (i.e., experience of empathy with protagonists in a film or a novel). To compare the scores obtained by Erica to the normative population, we computed $\mathrm{T}$ scores for each QCAE scales and subscales by using descriptive statistics for women, online administration retrieved from Di Girolamo et al. (2017). High scores on the QCAE usually indicate good empathy, whereas low scores indicate the lack of empathy. QCAE reported good alpha coefficients ranging from .70 to .85 (Gratz \& Roemer, 2004).

\section{Rorschach Performance Assessment System (R-PAS)}

The Rorschach test was administered, scored, and interpreted according to the Rorschach Performance Assessment System (R-PAS; Meyer, Viglione, Mihura, Erard, \& Erdberg, 2011) a new psychometrically sound evidence-based Rorschach method developed to overcome most of the limits of previous methods; a recent meta-analytic study by Mihura et al. (2013) proved the validity of the Rorschach variables and R-PAS (e.g., Pignolo et al., 2017). The Rorschach test is a performance-based test including 10 ambiguous, inkblot designs. The task is for the examinee to look at the inkblot and to answer to the question "What might this be?". The answers to this task are then coded based on i) what the examinee sees, ii) what, in the inkblot, made he or she see what he or she saw, and iii) where in the inkblot he or she was looking at. R-PAS raw scores may be converted into easyto-use, normalized standard scores (SS), which have a mean of 100 and standard deviation of 15 . According to the R-PAS manual, standard scores may be interpretable and potentially clinically relevant when Page 1 scores are at or below a standard score of about 90 or when they are at or above a standard score of about 110, and when Page 2 scores are at or below a standard score of about 85 or when they are at or above a standard score of about 115 . Variables included in Page 1 have empirical support higher than those in Page 2, so when the values of the latter are in disagreement with those on Page 1, they should be interpreted with caution, especially when their standard scores are not extremely elevated. Given that some R-PAS variables are related to the Complexity, R-PAS also offers Complexity Adjusted scores, so that clinicians may interpret the profile as if the examinee had obtained a typical level of complexity. In other words, because protocols with a high level of complexity may show elevations on multiple scores, Complexity Adjusted scores show what the person's protocol would look like when complexity is set to an average level. Five interpretative domains are included into the R-PAS: Administration Behaviors \& Observations, Engagement \& Cognitive Processing, Perception \& Thinking Problems, Stress \& Distress and Self \& Other Representation domains.

\section{Electrodermal activity measurement}

Before starting the experimental session Erica was welcomed in temperature and humidity controlled rooms $\left(\sim 18-22^{\circ} \mathrm{C}\right.$; humidity not higher than $\left.50 \%\right)$ in order to avoid any effects of temperature and humidity on EDA (Boucsein, 1992). Upon arrival, she was invited to seat in an armchair with comfortable headrest, arms, and back. After following standard, skin-cleaning procedures (Fowles, Christie, \& Edelberg, 1981; Schmidt \& Walach, 2000), EDA was measured by applying $\mathrm{Ag}-\mathrm{AgCl}$ electrodes on the distal (first) phalanges of the index and middle fingers of the participant's dominant hand. Electrical signal, more in detail, was recorded on line using Psycholab VD13S system (Satem, Rome, Italy) interfaced to a portable computer via Ethernet cable and with Psycholab P.C. Software (Operating system-Windows XP). 
It was acquired in micro-Siemens $(\mu \mathrm{S})$ at a sampling rate of $100 \mathrm{~Hz}$, via constant voltage method $(0.5 \mathrm{~V})$.

The skin conductance recording session was composed by three different steps: baseline, stress and recovery conditions. Specifically, during baseline, she was asked to rest and relax quietly for a period of 6 minutes while sitting. Immediately after the resting period, a 6 minutes stress-inducing task was started (stress), using the Mental Arithmetic Task (Dickerson \& Kemeny, 2004) (this method was largely used in previous psychophysiological studies: e.g., Condren, O'Neill, Ryan, Barrett, \& Thakore, 2002; Earle, Wolfgang, \& Weinberg, 1999; Kirschbaum et al., 1995; Lai \& Linden, 1992) a task involved a performance of serial subtraction, associated to discouraging feedback (sometimes called harassment) from the experimenter (see Giromini et al., 2016a, 2016b, for details). Soon after the stress-inducing task, a 6 minutes recovery period (recovery), was recorded during which Erica was requested to rest quietly. In order to evaluate in Erica sympathetic reactivity to stress, our statistical analyses focused on the EDA changes from baseline to stress and recovery. The procedure we adopted was consistent with previous EDA studies involving baseline, stress, and/or recovery conditions (e.g., Giromini et al., 2016a, 2016b; Healey \& Picard, 2005).

Psychophysiological studies examined in depth the association between hyperhidrosis with EDA and heart rate (HR) that reflect the autonomic nervous system (ANS) activity in response to stress, and emotional circumstances (e.g., Birner, Heinzl, Schindl, Pumprla, \& Schnider, 2000; Tronstad, Gjein, Grimnes, Martinsen, \& Krogstad, 2008). We expected that during the exposure to this type of laboratory stress-inducing task, EDA values increased from baseline to stress and decreased from stress to recovery showing that sympathetic arousal was activated by the induced stress (Boucsein, 1992, 2012).

\section{Magnetic resonance imaging acquisition}

Neuroimaging data were collected with a 3T Philips Ingenia scanner. Structural images of the whole brain were acquired using a $\mathrm{T} 1$ and a T2-weighted sequence (TR $8.1 \mathrm{~ms}$, TI $900 \mathrm{~ms}$, TE $3.7 \mathrm{~ms}$, voxel size $1 \times 1 \times 1$ $\mathrm{mm} 3$ ). Given that our study aimed to focus on primary hyperhidrosis, we used MRI in order to exclude the hyperhidrosis secondary to neurological problems. For example, excessive sweating has been reported in many lesions of the central nervous system, in brainstem infarction, and as related to signal changes that occurred on right hypothalamus (Appelenzer, 1982; Edwards, Lennox, Robson, \& Whiteley, 1996; Mahendiran al., 2009).

\section{Results}

\section{Magnetic resonance imaging exam and electrodermal activity measures}

MRI exam does not show clinical alterations in the brain. EDA measures are obtained for each of three phases during the experimental session: baseline: $M=27.68 \mu$, $S D=5.03 \mu$; stress: $\mathrm{M}=34.08, \mu$; $\mathrm{SD}=2.52 \mu$; recovery: $\mathrm{M}=22.45 \mu ; \mathrm{SD}=7.15 \mu$. This physiological profile indicates higher values than normal people; in fact, the normal baseline value of skin conductance is around $11.48 \pm 7.69$ $\mu$, while her value is $27.68 \mu$ (Kaushik, Mahajan, Rajesh, $\&$ Kaushik, 2004). This results means that Erica shows a hyperactive functioning of autonomic nervous system (e.g., Boucsein, 1992, 2012).

\section{Symptoms checklist-90-R}

The questionnaire reports $\mathrm{T}$ scores lower than 65 for all scales indicating the absence of overt psychopathology (Table 1).

\section{State-trait anxiety inventory}

Raw score of 36 and $\mathrm{T}$ score of 47 indicate the absence of symptoms for the state anxiety. In fact, a raw score of $39-40$ has been suggested to detect clinically significant symptoms for the State-Anxiety scale. Furthermore, other studies have suggested a higher raw score of 54-55 for older adults (Table 1).

\section{Difficulties in emotion regulation scale}

The scores Erica obtained on the Non-Acceptance, Goals, Impulse, and Strategies scales indicate respectively the tendency to accept negative emotions, capacities to perform a goal-directed behavior, abilities to control impulsive behaviors and to use of situationally appropriate strategies to modulate own emotional responses (Table 1). Furthermore, it is noteworthy that Erica obtained very low scores (i.e., lower than $30 \mathrm{~T}$ ) on the Awareness and Clarity scales indicating high knowledge and clarity of emotions and how they are experienced (Table 1).

\section{Toronto alexithymia scale}

The total score on TAS 20 is less than 51 (TAS Total Score $=22$ ) reporting non-alexithymia characteristics (e.g., Bagby, Parker, \& Taylor, 1994; Table 1).

\section{Questionnaire of cognitive and affective empathy}

A total score of the QCAE of $63 \mathrm{~T}$ indicates a good empathy capability. More in detail, the Cognitive Empathy scale score $(T=63)$ is higher than the Affective Empathy scale score $(\mathrm{T}=58)$, with the scores of the two subscales of the Cognitive Empathy (i.e., Perspective Taking and the Online Simulation) being about $60 \mathrm{~T}$, and the Peripheral Responsivity subscale being the highest $(\mathrm{T}=64)$. In general, these scores indicate that Erica's cognitive empathy skills are well developed and that her affective empathy skills are less effective when Erica is close to an empathy target (Table 1). In other words, it seems that the more Erica is exposed to others emotional states, the most she is emotional buried. 


\section{Rorschach performance assessment system}

Erica's performance during the exposure to the Rorschach test is reported below (Table 2). In order to make this paragraph understandable both to R-PAS experts and for those who are not familiar with such tool, the paragraph is organized as follows: the first part (i.e.,

Table 1. Self-reports.

\begin{tabular}{|c|c|c|}
\hline & & \\
\hline & Raw & $\mathbf{T}$ \\
\hline Symptoms Checklist-90-R & & \\
\hline Somatization (SOM) & 0.25 & 41 \\
\hline Obsessive-Compulsive (O-C) & 0.60 & 44 \\
\hline Interpersonal Sensitivity (I-S) & 0.44 & 44 \\
\hline Depression (DEP) & 0.38 & 43 \\
\hline Anxiety (ANX) & 0.60 & 48 \\
\hline Hostility (HOS) & 0.17 & 42 \\
\hline Phobic Anxiety (PHOB) & 0.00 & 44 \\
\hline Paranoid Ideation (PAR) & 0.50 & 44 \\
\hline Psychoticism (PSY) & 0.00 & 42 \\
\hline Global Severity Index (GSI) & 0.34 & 42 \\
\hline Positive Symptom Distress Index (PSDI) & 1.15 & 39 \\
\hline Positive Symptom Total (PST) & 27 & 44 \\
\hline State-Trait Anxiety Inventory & & \\
\hline Total & 36 & 47 \\
\hline Toronto Alexithymia Scale & & 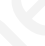 \\
\hline Difficulty Identifying Feeling (F1) & 8 & - \\
\hline Difficulty Describing Feelings (F2) & 5 & - \\
\hline Externally-Oriented Thinking (F3) & 9 & - \\
\hline Total & 22 & - \\
\hline Difficulties in Emotion Regulation Scale ${ }^{\mathrm{a}}$ & & \\
\hline Non-Acceptance & 14 & 52 \\
\hline Goals & 14 & 48 \\
\hline$\sqrt{2}$ & 10 & 44 \\
\hline Awareness & 6 & 29 \\
\hline Strategies & 16 & 48 \\
\hline Clarity & 5 & 27 \\
\hline Total & 65 & 34 \\
\hline Questionnaire of Cognitive and Affective & & \\
\hline Cognitive Empathy & 69 & 63 \\
\hline Perspective taking & 37 & 62 \\
\hline Online simulation & 32 & 60 \\
\hline Affective Empathy & 41 & 58 \\
\hline Emotion contagion & 11 & 49 \\
\hline Proximal responsivity & 14 & 55 \\
\hline Peripheral responsivity & 16 & 64 \\
\hline Total & 110 & 63 \\
\hline
\end{tabular}

${ }^{\mathrm{a}} \mathrm{T}$ scores computed by using equations reported by Giromini et al. (2017). ${ }^{\mathrm{b}} \mathrm{T}$ scores computed by using descriptive statistics for women, online administration retrieved from $\mathrm{Di}$ Girolamo et al. (2017).
Domains) includes specific clarifications that can be useful in approaching the interpretation of the more relevant variables in Erica's protocol; subsequently, the specific interpretation of the Erica's protocol is presented in the second part (i.e., Interpretation). At the end, a brief summary is reported. The interpretation of R-PAS protocol is split in five domains. The next paragraph includes the description of the R-PAS domains and includes the most significant variables of Erica's Rorschach protocol.

\section{Domains}

\section{Administration behavior and observations}

This cluster assesses basic task-relevant behaviors (for example, Erica held weakly the Rorschach cards, seeming hesitant to touch them). About this cluster, the most important variable for Erica is the Card Turning (CT); it is scored when the examinee turns the card in a different position from the one in which the card has been given. CT is related to intellectual curiosity, flexibility or defiance and anxiety.

\section{Engagement and cognitive processing}

The variables in this cluster are related to the individual's productivity, psychological resources, motivation and engagement in the test process. In particular, Complexity is comparable to the g-factor on an IQ test or Welsh's A on the MMPI-2. It measures differentiation, integration, and productivity that examinee provides during the task: a high Complexity index, along with Number of Responses and Blends, means that the person has brought a considerable amount of psychological activity and effort to cope with the demands required by the test. Noteworthy is the $(C F+C)$ Sum $C$ value, which is calculated basing on the dominance of color over form in determining the percept, and is a measure about how much is present the cognitive control and the modulation in one's reactions to the environment, especially when there is emotional provocation. When this score is low, as in this case, reactions to emotionally-toned stimuli tend to be modulated or muted by mental processing and cognitive control. Moreover, the $F D$ variable is coded when the shape of the inkblot suggests to the subject a view in three dimensions; high score in this code involve taking distance from something disturbing (Meyer et al., 2011).

\section{Perception and thinking problems}

This domain largely represents problems in thinking, judgment, or perception. Some variables involve conventionality and seeing the world as others do, represented by the $F-\%$ code. In particular, the EII-3 and the TP-Comp are related with the most severe psychological dysfunctions as reality testing, thought disorganization, crude and disturbing thought content. The first one, which is a measure of how much the Ego is damaged or healthy, is more related with traumatic and stressful situations or relation- 
Table 2. Rorschach (R-PAS) standard and complexity-adjusted scores.

\begin{tabular}{|c|c|c|c|c|c|c|c|c|c|c|c|}
\hline \multirow[t]{2}{*}{ Variable } & \multirow[t]{2}{*}{$\begin{array}{l}\text { Raw } \\
\text { Scores }\end{array}$} & \multicolumn{2}{|c|}{$\begin{array}{c}\text { Standard } \\
\text { Scores (SS) }\end{array}$} & \multicolumn{2}{|c|}{$\begin{array}{l}\text { Complexity } \\
\text { Adjusted } \\
\text { Scores }\end{array}$} & \multirow[t]{2}{*}{ Variable } & \multirow[t]{2}{*}{$\begin{array}{c}\text { Raw } \\
\text { Scores }\end{array}$} & \multicolumn{2}{|c|}{$\begin{array}{l}\text { Standard } \\
\text { Scores (SS) }\end{array}$} & \multicolumn{2}{|c|}{$\begin{array}{c}\text { Complexity } \\
\text { Adjusted } \\
\text { Scores }\end{array}$} \\
\hline & & $\%$ ile & Score & $\%$ ile & Score & & & $\%$ ile & Score & $\%$ ile & Score \\
\hline Page 1 & & & & & & Page 2 & & & & & \\
\hline \multicolumn{6}{|c|}{ Administration Behaviors \& Observations } & \multicolumn{6}{|c|}{ Engagement \& Cognitive Processing } \\
\hline Pr & 1 & 62 & 104 & & & $\mathrm{~W} \%$ & $46 \%$ & 63 & 105 & 58 & 103 \\
\hline $\mathrm{Pu}$ & 0 & 40 & 96 & & & $\mathrm{Dd} \%$ & $23 \%$ & 77 & 111 & 79 & 112 \\
\hline CT & 0 & 18 & 86 & & & SI & 7 & 97 & 127 & 97 & 127 \\
\hline \multicolumn{6}{|c|}{ Engagement \& Cognitive Processing } & IntCont & 4 & 79 & 112 & 74 & 110 \\
\hline Complexity & 84 & 69 & 107 & & & $\mathrm{Vg} \%$ & $0 \%$ & 18 & 86 & 18 & 86 \\
\hline $\mathrm{R}$ & 26 & 69 & 107 & 55 & 102 & $\mathrm{~V}$ & 0 & 29 & 92 & 29 & 92 \\
\hline $\mathrm{F} \%$ & $35 \%$ & 36 & 95 & 44 & 97 & FD & 4 & 97 & 129 & 97 & 129 \\
\hline Blend & 4 & 56 & 102 & 37 & 95 & $\mathrm{R} 8910 \%$ & $35 \%$ & 72 & 109 & 73 & 109 \\
\hline Sy & 8 & 64 & 106 & 52 & 101 & WSumC & 1.5 & 21 & 88 & 10 & 81 \\
\hline $\mathrm{MC}^{\mathrm{a}}$ & 7.5 & 57 & 103 & 39 & 96 & $\mathrm{C}$ & 0 & 36 & 95 & 36 & 95 \\
\hline MC - PPD & -0.5 & 64 & 105 & 64 & 105 & $\mathrm{Mp} /(\mathrm{Ma}+\mathrm{Mp})$ & $50 \%$ & 61 & 104 & 61 & 104 \\
\hline $\mathbf{M}$ & 6 & 82 & 113 & 67 & 107 & \multicolumn{6}{|c|}{ Perception \& Thinking Problems } \\
\hline $\mathbf{M} / \mathbf{M C}$ & $80 \%$ & 91 & 120 & 91 & 120 & $\mathrm{FQu} \%$ & $35 \%$ & 69 & 107 & 70 & 107 \\
\hline$(\mathrm{CF}+\mathrm{C}) / \mathrm{SumC}$ & $0 \%$ & 5 & 75 & 5 & 75 & Stress \& Distress & 8 & & & & \\
\hline \multicolumn{6}{|c|}{ Perception \& Thinking Problems } & PPD & 8 & 42 & 97 & 29 & 92 \\
\hline EII-3 & 0.6 & 84 & 115 & 85 & 116 & CBlend & 0 & 28 & 91 & 28 & 91 \\
\hline TP-Comp & 1.2 & 78 & 111 & 78 & 111 & $\mathrm{C}^{\prime}$ & 1 & 41 & 97 & 23 & 88 \\
\hline WSumCog & 10 & 70 & 108 & 66 & 106 & $\mathrm{~V}$ & 0 & 29 & 92 & 29 & 92 \\
\hline SevCog & 1 & 80 & 113 & 80 & 113 & CritCont $\%$ & $27 \%$ & 73 & 109 & 71 & 108 \\
\hline FQ-\% & $15 \%$ & 77 & 111 & 77 & 111 & \multicolumn{6}{|c|}{ Self \& Other Representation } \\
\hline WD-\% & $10 \%$ & 65 & 106 & 57 & 103 & SumH & 9 & 81 & 113 & 75 & 110 \\
\hline FQo $\%$ & $50 \%$ & 25 & 90 & 21 & 88 & NPH/SumH & $89 \%$ & 93 & 122 & 93 & 122 \\
\hline $\mathbf{P}$ & 6 & 59 & 103 & 63 & 105 & V-Comp & 5.7 & 94 & 123 & 92 & 121 \\
\hline Stress \& Distress & $\mathrm{r}$ & 0 & 36 & 95 & 36 & 95 & & & & & \\
\hline YTVC' & 3 & 36 & 95 & 23 & 89 & $\mathrm{p} /(\mathrm{a}+\mathrm{p})$ & $55 \%$ & 74 & 110 & 74 & 109 \\
\hline $\mathbf{m}$ & 0 & 14 & 84 & 14 & 84 & AGM & 1 & 75 & 110 & 75 & 110 \\
\hline $\mathrm{Y}$ & 0 & 17 & 85 & 17 & 85 & $\mathbf{T}$ & 2 & 89 & 118 & 89 & 118 \\
\hline MOR & 4 & 94 & 123 & 92 & 121 & PER & 1 & 72 & 109 & 72 & 109 \\
\hline SC-Comp & 4.6 & 55 & 102 & 43 & 97 & An & 2 & 71 & 108 & 71 & 108 \\
\hline
\end{tabular}

Self \& Other Representation

\begin{tabular}{lccccc}
\hline ODL $\%$ & $4 \%$ & $\mathbf{1 3}$ & $\mathbf{8 3}$ & $\mathbf{9}$ & $\mathbf{8 0}$ \\
\hline SR & 1 & 56 & 102 & 56 & 102 \\
\hline MAP/MAHP & $33 \%$ & 29 & 92 & 28 & 91 \\
\hline PHR/GPHR & $36 \%$ & 58 & 103 & 58 & 103 \\
\hline M- & 1 & $\mathbf{8 1}$ & $\mathbf{1 1 3}$ & $\mathbf{8 1}$ & $\mathbf{1 1 3}$ \\
\hline AGC & 10 & $>\mathbf{9 9}$ & $\mathbf{1 4 3}$ & $>\mathbf{9 9}$ & $\mathbf{1 4 3}$ \\
\hline H & 1 & $\mathbf{2 1}$ & $\mathbf{8 8}$ & $\mathbf{9}$ & $\mathbf{7 8}$ \\
\hline COP & 3 & $\mathbf{9 1}$ & $\mathbf{1 2 0}$ & $\mathbf{9 0}$ & $\mathbf{1 1 9}$ \\
\hline MAH & 2 & $\mathbf{8 6}$ & $\mathbf{1 1 6}$ & $\mathbf{7 0}$ & $\mathbf{1 0 8}$
\end{tabular}

Pr, Prompt; Pu, Pull; CT, Card Turns; R, Number of responses; F\%, Percentage of pure form responses; Sy, Synthesis; MC, Human Movement and Weighted Color; MC-PPD, Human Movement and Weighted Color minus Potentially Problematic Determinants; M, Human Movement; M/MC, Human Movement Proportion; (CF+C)/SumC, Color Dominance Proportion; EII-3, Ego Impairment Index-3; TP-Comp, Thought \& Perception Composite; WSumCog, Weighted Sum of the Six Cognitive Codes; SevCog, Severe Cognitive Codes; FQ- $\%$, Percentage of FQ- responses; WD-\%, Percentage of FQ- in Whole and Details localization; FQo\%, Percentage of FQo responses; P, Popular responses; YTVC', Sum of Shading and Achromatic Color; m, Inanimate Movement; Y, Diffuse Shading; MOR, Morbid Content; SC-Comp, Suicide Concern Composite; ODL\%, Percentage of responses with Oral Dependency Language; SR, Space Reversal; MAP/MAHP, MAP proportion; PHR/GPHR, PHR Proportion, M-, Human Movement with FQ-; AGC, Aggressive Content; V-Comp, Vigilance Composite; H, Human Content; COP, Cooperative Movement; MAH, Mutuality of Autonomy Health; W\%, Percentage of W responses; Dd\%, percentage of Dd responses; SI, Space Integration; IntCont, Intellectualized Content; Vg\%, Percentage of Vg responses; V, Vista; FD, Form Dimension; R8910\%, Percentage of responses to cards 8, 9, 10; WSumC, Weighted Sum of Color; C, Color; Mp/(Mp+Ma), Mp Proportion; FQu\%, Percentage of FQu responses; CBlend, Color Blended with Shading and Achromatic Color; C', Achromatic Color; CritCont\%, Critical Contents; SumH, All Human Content; NPH/SumH, Non-Pure H Proportion; r, Reflections; p/(p+a), Passive Proportion; AGM, Aggressive Movement; T, Texture; PER, Personal Knowledge Justification; An, Anatomy. 
ships; the second one is usually high in psychotic functioning or could be moderately severe disorders without clear psychosis, depending on one of the two components (Meyer et al., 2011).

\section{Stress and distress}

The variables in this domain have strong relationships to stress and distress in various forms. The most problematic variable in Erica's protocol is the MOR, which is usually coded when the percept is provided as damaged, flawed, or somehow harmed by life and external events. High scores would suggest a dysphoric and negative view of the world and the presence of implicit distress (Meyer et al., 2011).

\section{Self and other representation}

All these variables are related to ways of understanding self, others, and relationships as a foundation of interpersonal relatedness. The elevated number of aggressive contents, represented by the AGC variable, reflects aggressive concerns, preoccupations, and/or identifications. The most interesting variables in this protocol are the $T$ and the V-Comp. The first one is coded when shades of the inkblot suggest a tactile sensation and represents a measure of sensitivity to tactile impressions that is thought to translate to interest in interpersonal closeness or contact. The second one is and index of guardedness, focused cognition, sensitivity to cues of danger, and interpersonal wariness or distancing.

\section{Interpretation}

\section{Administration behavior and observations}

Erica holds weakly the Rorschach cards, seeming hesitant to touch them; however, her productivity is in the average range (Prompt $[\mathrm{Pr}]=104$; Pull $[\mathrm{Pu}]=96$; Number of Responses $[R]=107)$ suggesting that Erica follows the instructions to give two, or maybe three, responses per card (Meyer et al., 2011). Interestingly, Erica never turns the card, which is less frequent compared to the norms. Usually, this behavior would be interpreted as a hesitancy to interact with the environment and to manipulate it, or a high level of compliance to the circumstances or to the instructions that were given. However, in this specific case this behavior may be consistent with the way Erica holds the cards, as she is visibly uncomfortable in touching them given her illness cards.

\section{Engagement and cognitive processing}

Erica seems to have adequate level of cognitive processing, productivity, psychological resources, and engagement to the world. Erica does not execute the task carelessly (Complexity=107 SS; Number of Responses $[R]=107 \mathrm{SS}$ ) and shows good capability in structuring perception in different ways (Blends $[\mathrm{Bl}]=102 \mathrm{SS}$ ), to take into account the whole situation, and using all of the available information from the environment (Percentage of whole [W\%]=105 SS). Moreover, Erica shows good capability to synthetize and to integrate different perceptions and ideas in a whole view (percentage of Form [F\%] $=95$ SS; Synthesis $[\mathrm{Sy}]=106 \mathrm{SS}$ ) without being overwhelmed by uncommon, small, or idiosyncratic details (percentage of Uncommon Detail [Dd\%]=111 SS). She reports a sophisticated cognitive processing and a high capability to evaluate, differentiate, and integrate with flexibility and creativity features of the environment (Space Integration $[\mathrm{SI}]=127 \mathrm{SS}$ ). Indeed, these different facets of the environment do not collapse in a vague or impressionistic cognitive style (Percentage of Vague [Vg\%] $=86 \mathrm{SS}$ ).

Erica's inner world is full, multifaceted, and characterized by elements that could be both resources and barriers at the same time; however, there is a good balance regarding passive/receptive versus active/goal-directed thinking (Proportion of Passive Human movement responses $[\mathrm{Mp} /(\mathrm{Ma}+\mathrm{Mp})]=104 \mathrm{SS})$. In general, Erica's level of reactivity and vitality is in the average range (Human Movement and weighted sum of Color Determinants and Potential Problematic Determinants [MCPPD $=105 \mathrm{SS}$; weighted sum of Color Determinants [WSumC=88 SS]; Pure Color determinant $[\mathrm{C}]=95 \mathrm{SS}$ ), suggesting that she is as responsive as most people when exposed to vibrant stimuli or provocative situations (Number of responses at card VIII, IX, X [R8910\%] $=109$ SS). On the other hand, this inner vivacity could have some difficulties in coming out freely. In fact, Erica shows a strong tendency to reflect and reason rather than reacting spontaneously to emotions, impulses, or circumstances (Human Movement proportion $[\mathrm{M} / \mathrm{MC}]=120 \mathrm{SS}$ ). This tendency is also shown by an excessive control over and the presence of an affective constriction in response to strong emotional and environmental stimuli (The Color Dominance Proportion $[(\mathrm{CF}+\mathrm{C}) / \mathrm{SumC}]=75 \mathrm{SS})$, and by the tendency to use intellectual strategies (Intellectualized Content [IntCont] $=112 \mathrm{SS}$ ).

Erica tends to avoid to living in the moment - especially when this implies being involved in external events - and prefers to live in embellished and imaginative inner world. Likewise, this capability in disengagement (e.g., in cases of looming circumstances or stressing events) could become an actual defensive strategy, as some kind of dissociation mechanism, in order to protect Erica from her insecurity (Form Dimension [FD]=129 SS); FD variable is traditionally related to the perspective-taking process and to the keeping of distances from the environment: indeed, in case of depression symptoms, FD might be interpreted as a negative self-view. She seems to be a reflective person, who is strongly able to mentalize and to experiment the self as the main agent of her own experiences (Human Movement $[\mathrm{M}]=113 \mathrm{SS}$ ). However, in order to better understand the meaning of this index, it is necessary to look at the Human Movement Form Quality 
Minus (M-) score (see below, in Self and others representation section).

\section{Perception and thinking problems}

Both the Ego Impairment Index and Thought (EII $3=115$ SS) and the Thought \& Perception Composite (TPComp=111 SS) score are above the average. EII suggests that Erica may have personality dysfunction, chronic stressors, or trauma, while TP-Comp could indicate that she might have some difficulties in thinking clearly and seeing things accurately. Although Erica shows an average capability to interpret the environment in a usual and widely accepted way (Weighted Sum of the Cognitive Codes [WSumCog] $=108$ SS; Percentage of Formal Quality Ordinary $[\mathrm{FQo} \%]=90 \mathrm{SS}$; Popular responses $[\mathrm{P}]=103$ SS; Percentage of Formal Quality Unusual [FQu\%] $=107$ SS), idiosyncratic, unique or individualized ways of perceiving events sometimes interfere with the accuracy in the perception of the external environment (Severe Cognitive codes $[\mathrm{SevCog}]=113 \mathrm{SS}$; percentage of Formal Quality Minus [FQ-\%]=111 SS). However, these distortions occur likely just when there is something that interferes with her usual strategies, or when she is involved in an uncommon, unfamiliar and unconventional environment (percentage of Whole and common Detail Minus [WD-\%]=106 SS). In conclusion, it seems that Erica is able to see things as other people do, and that she has an adequate reality testing. However, when she is exposed to themes that are highly painful to her, her cognitive functioning may also have severe slippage.

\section{Stress and distress}

Overall, Erica does not report high levels of distress related to anxiousness, irritation, and dysphoria or distracting ideation associated with environmental stressors that she may feel outside of her control, or determined by external forces (Shading and Achromatic Color $\left[\mathrm{YTVC}^{\prime}\right]=95 \mathrm{SS}$; Diffuse Shading codes $[\mathrm{Y}]=85$ SS; $\mathrm{m}=84 \mathrm{SS}$ ). Moreover, she is not subjected to disruptive or irritating internal demands (Potential Problematic Determinants $[\mathrm{PPD}]=97 \mathrm{SS}$ ), emotional or environmental sensitivity (Color Blended with Shading or Achromatic Color [CBlend] $=91$ SS), gloomy, dark, and dreary stimuli (Achromatic Color [C']=97 SS), and worries characterized by primitive mental imagery or traumatic experiences (Critical Content [CritCont] $=109$ SS). However, Erica reports a high number of morbid images (Morbid Content $[\mathrm{MOR}]=123 \mathrm{SS}$ ), which may indicate the presence of themes of damage characterizing her life. Indeed, the Morbid score is a thematic code that involves damage, death, or dysphoria and it is significantly related to the presence of morbid thoughts as maltreatments, chronic pain, traumatic experiences, and major depression (Mihura, Meyer, Dumitrascu, \& Bombel, 2013). Since other depression indices (CBlends, C', PPD, YTVC') are characterized by significant thresholds, the high Morbid score might represent a view of herself as damaged, flawed or harmed by her life suggesting that a form of serious implicit stress may be present, even if there is not a severe depressive condition.

\section{Self and other representation}

In general, she is not self-centered (Reflex $[\mathrm{r}]=95 \mathrm{SS}$ ): Erica is able to envision mature and mutually enhancing interactions respecting the independence and separateness of the participants (Proportion of Mutuality of Autonomy scores [MAP/MAHP] $=92 \mathrm{SS}$; Mutuality of Autonomy Health $[\mathrm{MAH}]=116 \mathrm{SS}$ ), and her attitude to have an active or a passive role in relational situations seems balanced (Passive Proportion $[\mathrm{p} /(\mathrm{a}+\mathrm{p})]=110 \mathrm{SS})$. Her ability to understand herself and others in an adaptive way is in the average (Human Representation Proportion $[$ PHR/GPHR] $=103$ SS): she does not have evidence of notable implicit dependency needs (Oral Dependency Language $[\mathrm{ODL}]=83 \mathrm{SS}$ ) or propensity for oppositionality attitudes and behaviors (Space Reversal [SR]=102 SS). Moreover, she does not abuse of defensive self-justifications (Personal Knowledge Justification [PER]=109 SS) and, unexpectedly, she does not have more concerns about the body integrity or illness than most people (Anatomy Content $[\mathrm{An}]=108 \mathrm{SS})$.

\section{Summarizing}

Erica presents many cognitive and psychological resources that make her compliant, engaged with the world, able to synthetize and to integrate big and small details in an integrated view. This comprehensive view includes a flexible and creative way to understand the environment.

As regard the human interactions, Erica is able to respect the independence and separateness of the participants and shows interest and attentiveness to others. This capability and this tendency to read others intentions could become a sort of vigilant watchfulness (All Human Content $[\mathrm{SumH}]=113 \mathrm{SS}$ ) in those situations in which there is a high level of emotional closeness and relational intimacy. This guarded and focused style of processing information is likely associated with interpersonal distancing and wariness (Vigilance Composite [VComp]=123 SS; see FD in Engagement and cognitive processing) that, in this case, hides a desire and a capacity for closeness and contact with others. V-Comp indicates the tendency to be chronic activated in order to protect oneself from the environment that is perceived as potentially dangerous (Meyer et al., 2011). However, she expressed a strong desire of interpersonal closeness and contact, which may be associated with a feeling of loneliness that can show unmet interpersonal needs (Texture $[\mathrm{T}]=118 \mathrm{SS}$ ). On the other hand, Erica has a propensity to view relationships as supportive and helpful, or at least to seek out this kind of relations (Cooperation Movement $[\mathrm{COP}]=120 \mathrm{SS}$ ). Indeed, in two of the three COP responses, the COP code is in an unrealistic context. The 
$19^{\text {th }}$ response is described below. The other one (i.e., $4^{\text {th }}$ response) contains two aggressive creatures involved in a Fabulate Combination (FAB) - "two rhino cubs kissing". In general, it is possible that the Erica's representation of relationships as supportive and helpful is present, but it could be more in her imagination that in her daily life. More specifically, the possibility to find relief and aid in her significant others it is more desired than experienced.

The other response where the COP is present is in a realistic context, with an accurate Formal Quality, and with real and entire human figures: "Two human figures who are doing something in a basin. [...] Two female figures, because it seems the visage, the neck, the breast, a bit protruding nose... and the hands as if they were washing the clothes or they were washing hands in the basin. And it seems that they had heels". This response shows a possibility that helpful relationships could exist in reality. The theme of water is present and abundant, and the scene describes the gesture of cooperation about washing something - probably the hands - with someone else. This scene reminds the scene described by Erica about when her mother used to wash her hands when she was a baby. However, after this good response, Erica needs to be prompt to continue the task, with the occurrence of the first cognitive slippage in the subsequent response. Perhaps, this identification caused a sort of broke down of her concentration.

Moreover, Erica seems to be characterized by the inclination to represent herself and others in an incomplete, unrealistic, and fanciful ways ( $\mathrm{H}=88 \mathrm{SS}$; Non-Pure H Proportion $[\mathrm{NPH} / \mathrm{SumH}]=122 \mathrm{SS}$ ), being more interested in imaginary characters than in the real people. As mentioned above this result is confirmed by the $\mathrm{M}$ - score (Human Movement Minus [M-]=113 SS), which suggests that her mentalization capability may collapse in a misperception of human images or activity. Erica's preoccupations may interfere with the realistic appraisal and understanding of other people thoughts and intentions. Indeed, the most severe cognitive slippage occurred right after the exposure to the colored cards (i.e. the $18^{\text {th }}$ response), and her thought capabilities seem to be still low at the subsequent response (i.e. the $19^{\text {th }}$ response) where there is the only one M- code: "two figures, as if they were two mermaids that are touching each other with the arms on the top. [...]. It reminds me two female figures, but here it is as if they have a tail, thus two mermaids. They are touching each other as if they were get out of the water to do those shows". In this response, several Erica's life topics are present. The human figure is split in two, the upper part is in contact with the other figure, while the lower part is a fishtail, namely a not humanlike part typical of water-creatures. A possible explanation could be that the colors have aroused Erica's emotional world, thus Erica made a big effort in order to control this possible upsetting situation, and few cognitive resources remained at her disposal to performance adequately the task. In addition, in this response, the theme of the contact is well represented. From what Erica said, her feet are the tool through which she controls her hyperhidrosis: when she is not wearing shoes, her hands do not sweat, and a direct contact with the external world is feasible. Indeed, this is the only response in the entire protocol with a contact between two human figures, and the frame is an unreal dimension. The temporary failure of her cognitive defenses, combined with the emotional emerging, may cause the arising of her desire of contact with other people.

Finally, Erica is not a person with an attitude toward violent activity (Aggressive Movement [AGM] $=110 \mathrm{SS}$ ), however her level of identification with power, aggressiveness, and dangerousness is greater than the average range (Aggressive Content $[\mathrm{AGC}]=143 \mathrm{SS}$ ) and she may have concerns about aggressiveness without being an aggressive or violent person. Looking at the responses, the aggressive contents reported by Erica referred to animals' claws, hands that are potentially dangerous for human beings (i.e., wolf-like paws, crab's claws). Interestingly, these animal hands were not usually part of the animal described by Erica, determining the Incongruous Combination (INC) coding. Few examples are reported as follows: In the $18^{\text {th }}$ response, there is the most severe INC (INC2) "[...] two groundhogs, but with wolf-like paws, that are climbing on a ... as if it was a promontory, a mountain", while in the $22^{\text {nd }}$ response the less severe INC (INC1) "there is a crab, with touching claws. [...] Two claws, as if the crab was whitellight blue and the claws are orange... thus they shouldn't be its, because of the color". Given that the AGC variable has had a good support in the research literature as an indicator of aggressive, dangerous, and powerful images in the person's mind and that these hands were coded AGC, we may infer that these responses reflect Erica's representation of her hands as something dangerous to engage with.

\section{Discussion}

The different types of psychological and psychophysiological tools employed contributed to a composite and dynamic Erica's profile. Generally, self-reports did not report psychopathological symptoms in Erica. TAS showed that Erica did not present difficulties in identifying and describing one's own emotions exhibiting high emotional functioning associated to emotion recognition. As reported by SCL-90- R and STAI Y1, affective distress, worry or anxiety were absent. Erica seems not to feel discouraged, sad, hopeless, and unmotivated; she does not show psychopathological problems interfering her daily activities. Moreover, positive characteristics were reported by the DERS. Indeed, Erica showed high control and reduction of negative emotions, and in particular, by an elevated capacity to experience the full range of emotions. As reported by DERS, we can observe in Erica the understanding and acceptance of emotions, the ability to 
engage in goal-directed behaviors, and the adaptive use of situationally appropriate strategies especially in familiar and structured situations.

Erica shows the capacity to understand and resonate with the experiences of other people; as reported by QCAE, cognitive features of empathy seem to be associated to the capacity for taking another individual's perspective (i.e., mentalization ability) and affective empathy aspects are related to sharing the emotions of other people through observation or imagination of their experience. Specifically, we observed an emotional responsiveness to the feelings of others who are close within the social or affective subject's context.

As mentioned in the introduction section, stressful situation triggered increased activity in the sympathetic nervous system (SNS). In response to a stressor, SNS activity increase to mobilize metabolic energy for fight-orflight reaction (e.g., McEwen, 1998). Erica seems to be more subject to stress and distress and thus more prone to show exaggerated physiological reactions to stress, too (Meaney, 2001). In fact, Erica's physiological profile is characterized by higher values than normal people especially in the baseline condition. Because high values of EDA in baseline and EDA changes from baseline to stress resulted in sympathetic activity (Boucsein, 1992; IonescuTirgovişte \& Pruna, 1993; Mestanik, Visnovcova \& Tonhajzerova, 2014; Papez, 1937), by using the R-PAS we obtained important information on which way Erica may cope her difficulties related to her hyperhidrosis occurring during social and/or stressful condition. A, Erica seems to have empathic features more intense with characters in movies or novels characterized by fantastic relationships; this result is consistent with other findings obtained by the Rorschach test. Indeed, Erica seeks out a shelter in unrealistic or fantasized relations representing a unique way to manage her feeling of loneliness. Erica tends to avoid to live in the moment and prefers to live in embellished and imaginative inner world with a mild restriction in the range or intensity of display of feelings. She shows the tendency to use intellectualization strategies a defense mechanism that involves intellectualizing a stressful situation such that dealing with it becomes easier. Erica usually prefers to construct a fateful way in which it might possible to experience social situations and relationships without being overwhelmed by the discomforts. Her desire to establish relationship to the other people can be conditioned by hyperhidrosis, and the anxiety related to the illness affects her interpersonal intimacy conditions involving in detachment and withdrawal behaviors.

Moreover, we can observe in Erica a vigilant and wariness interpersonal style that limits her desire of spontaneity and authenticity in the interactions with people. Rorschach test reports the presence of feelings of damage or inadequacy, inferiority complex, and insecurity with a strong desire of interpersonal closeness. Nevertheless, Erica is characterized by adequate level of psychological resources and engagement in the world; she shows a sophisticated cognitive processing and a high ability to evaluate, differentiate, and integrate with flexibility and creativity several aspects of the environment.

The hyperhidrosis influences how Erica relates to others and, thus, her excessively sweaty palms can represent a debilitating and embarrassing daily condition: touching or holding hands is the first step toward experiencing that bonding feeling with someone else and, furthermore, the use of hand gestures may invalidate the powerful way when you communicate. For example, as reported by the CT Rorschach variable, we noted the hesitancy of Erica to interact with the environment or to manipulate it, and a behavior consistent with her appearing uncomfortable in touching the cards. Over time, Erica has tried to use specific cognitive strategies to address the problems caused by hyperhidrosis and to manage rationally the impact of negative emotions during daily life experiences. Erica has aimed to enhance her emotional competence, which includes awareness and clarity of emotional experience reflecting the attempt for responding to high distressing contexts such as social contexts (Saarni, 1999). Erica probably improved the clarity and understanding of her emotions in order to manage them and, thus, in order to control her hyperhidrosis, in the best way (see the scores of Clarity and Awareness scales of DERS).

We can infer that Erica fails in controlling negative emotions during stressful events (e.g., during the laboratory induced stress, or Rorschach test administration) and during unpredictable situations. In fact, we observed high levels of skin conductance reflecting an evident sympathetic reactivity. As reported by Gross and colleagues (1998), sometimes the attempts to suppress expressive behaviors during emotion-eliciting situations may lead to unpleasant experience and may increase sympathetic nervous system activation. Indeed, the attempts to influence ongoing mental processes may have paradoxical or unintended effects such as, for example, an increasing of sweating (Gross \& Levenson, 1997).

It is noteworthy that using R-PAS is possible to obtain both information related to the conscious awareness and observable behaviors (e.g., Card Turning; Prompt and Pull), and those not necessarily implicit/filtered through rational cognitive processes (e.g., $(C F+C)$ Sum $C$ related to reactions to emotionally-toned stimuli). Rorschach is a behavioral task that allows individuals to choose their responses whose behaviors implemented are an expression of their personality features and their style of processing the stimulus. The most valid clinic interpretations are those whose behaviors, codified within the microcosm of the test, can be generalized to verbal and perceptual behaviors similar to those implemented in the external environment. We can also speculate on the possible association between attachment system, psychophysiological arousal, and emotion regulation of Erica (it is noteworthy that EII-3 suggested that Erica had chronic 
stressors, or traumatic experiences). Several studies reported that ambivalent/preoccupied individuals for frequently enmeshing with their caregivers displayed elevated cardiovascular reactivity and skin conductance in response to stressful situation (e.g., Dozier \& Kobak, 1992; Roisman, 2007). As mentioned above, during childhood, Erica felt to be the focal point of the family because she often was involved in resolving the conflicts between her mother and father. She perceived that she would have had a great responsibility in resolving such hostile situation. Erica reports she experienced a painful occurrence while her parents were arguing each other. While being preoccupied for his father leaving his family, Erica was overwhelmed with anxiety and fear. We could hypothesize that the high stressful family situations contributed to increase the hyperhidrosis condition.

Overall, Erica seems to have lived in a family context characterized by uncertainties where it was not made explicit what daily happened (e.g., "He was often upset for wearing my socks, I do not know why"; "often my family made me anxious. I had not to talk with my parents about their marital conflicts; my parents pretended that everything was fine"). She had to understand her family relationships without directly talking on conflicts or difficulties regarding to her family members. Erica could not be a fragile or unstable person ("You mustn't cry! You have to stop him"). In such emotive chaos characterized by stress caused by things that were unsaid or were implicitly understood, over time she took distance from this perceived disturbing confusion and tended to avoid living in the moment, but to experience an imaginative world probably when she was alone, at home. She preferred not to stay in this enmeshed role within her family context, where she felt the focal point. Erica seems to use a defensive strategy in order to protect herself from the insecurity such as that emotive chaos (as reported by the FD Rorschach variable). Erica shows an emotional responsiveness to the feelings of others who are close within the affective subject's context; thus, when Erica is exposed to others emotional states, the most she is emotional buried (are noteworthy the QCAE scores, in particular those on Affective scale, and the V-Comp Rorschach variable). Whether Erica fails in keeping of distances from the environment and/or emotive chaos she may have some difficulties in thinking clearly and seeing things accurately (as reported, for example, by the TP-Comp Rorschach variable).

Furthermore, we can hypothesize that Erica escapes from her family and associated stress causing her excessive sweating by doing and performing several activities (e.g., climbing, martial arts, and play the guitar). Erica seems to be characterized by those coping strategies resulted in sport and dynamic activities associating with arousal control, relaxation, concentrating on goals, time management, increasing effort, wishful thinking, refocusing, information seeking, and maintaining positive focus, identifying those key insights and reaching conclusions from which you can benefit in the future (e.g., Naseem \& Khalid, 2010; Trapnell \& Campbell, 1999). Summarizing, Erica shows two main strategies in response to external environmental stimuli: excessive control and affective constriction (as reported by the color dominance proportion in the Rorschach and the Affective scale) with the use of intellectual strategies, and living in embellished and imaginative inner world in which may experience several feelings (as reported by the Engagement and Cognitive Processing Rorschach domain) or to do something concretely. These aforementioned aspects emerged from selfreports measures and the Rorschach test, but not from the physiological profiling (as we reported above, the suppression of expressive behaviors during emotion-eliciting situations may increase sympathetic nervous system activation). Thus, we can observe a substantial difference between the way in which Erica described herself (for example, DERS did not report high levels of stress) and which involuntary physiological changes occur that are beyond her rational control. Further details of Erica psychophysiological profile are discussed below.

\section{Conclusions}

As mentioned before, previous experimental suggested that an excessive response in sympathetic nerve activity to stressful stimuli was responsible for the intense and abundant sweating. Other studies reported that ambivalent/preoccupied individuals for frequently enmeshing with their caregivers displayed elevated cardiovascular reactivity and skin conductance in response to stressful situation; we speculated that Erica felt to be the focal point of the family because she often was involved in resolving the conflicts between her mother and father. Erica was overwhelmed with anxiety and fear. We might hypothesize that the high stressful family situations and traumatic experiences contributed to increase the hyperhidrosis condition. Therefore, Erica has tried to manage her emotional inner world and emotions to cope those stressful situations that might cause her excessive sweating. Overall, when some external contexts are perceived as potentially stressful but as predictable (e.g., staying at home with her relatives), Erica seems to use two cognitive strategies:

i) To seek refuge in a fantasy world where she may experience the full range of emotions that perhaps in the real world she could not live because of increase of her hyperhidrosis.

ii) To undertake dynamic activities associating with arousal control, relaxation, concentrating on goals and maintaining positive focus.

Differently, Erica appears to suffer from several difficulties during unpredictable situations showing high level of skin conductance reflecting an evident sympathetic reactivity; in fact, Erica may present problems in thinking 
clearly and seeing things accurately caused by feelings of embarrassment that in turn contribute to enhance her sweating. Therefore, we might wonder: What is that produces such vicious circle mentioned above? The vicious circle might be strongly associated with a maladaptive thought process as the rumination involving in difficulties in redirecting attention away from negative thoughts. We can suppose that a neurotic level organization may be present given that she seems to feel her problems arise from internal conflict as excessive; at the neurotic level, poorly adaptive defensive patterns are limited to an area of specific difficulty (Shapiro, 1965, 1981, 1989, 2002). For example, the defenses of patients characterized by neurotic characteristics, even when they are inflexible and problematic, are related to a specific area of psychological functioning: for example, in Erica we can observe problems especially in those situations perceived as potentially stressful because of their unpredictability. A metacognitive approach that deals with thoughts processes may make Erica aware of typical thought distortions and of how to change such thought distortions. From a metacognitive point of view, the psychotherapist should be work on following cognitive, attentional, and behavioral processes that may support the rumination thought: the excessive worry on excessive sweating, the focus of attention on own physiologic state, and the stable searching for possible early signs of hyperhidrosis during social and uncertain situations. Furthermore, a therapeutic approach should be able to provide Erica with a secure base from which to explore both herself and her relations with all those with whom she has made or might make an affectional bond (Bowlby, 1977). The therapist should help Erica to explore past and present attachments, including their expectations, feelings, and behaviors.

Our study has the merit to be the first to combine Rorschach data with physiological data in a hyperhidrotic case, and the use of psychophysiological methods provided additional elements of incremental validity to the assessment, engaging different processes. Indeed, most of the previous studied examined the effects of the hyperhidrosis disease on the psychological well-being in an individual but not investigating how specific personality features and cognitive strategies may be associated to the managing of a disabling problem such as that of hyperhidrosis. During the unstructured Rorschach task, Erica showed difficulties in managing those implicit aspects influencing the response processing: data obtained by the Rorschach are not relate exclusively to the conscious awareness providing essential information on the overall personality functioning.

One of possible limitations of this study is that specific tools able to evaluate the attachment style (e.g., the Adult Attachment Interview - AAI; George, Kaplan \& Main, 1996) were not included in assessing Erica. Indeed, given that insecure/anxious attachment may lead to the impaired emotion regulation (Gander \& Buchheim, 2015; Roisman,
2007), it would have been useful to investigate possible associations between the attachment style and the autonomic nervous system activity in response to stress. Although the most prominent critique of single case study is the issue of external validity or generalizability, our findings may improve the knowledge in understanding the functioning of personality where the hyperhidrosis occurs. Therefore, we chose to make a single case study in order to examine thoroughly those qualitative information related to psychological functioning that could not be obtained through a quantitative-multiple cases study.

\section{References}

Adar, R., Kurchin, A., Zweig, A., \& Mozes, M. (1977). Palmar hyperhidrosis and its surgical treatment: a report of 100 cases. Annals of Surgery, 186(1), 34.

Ak, M., Dincer, D., Haciomeroglu, B., Akarsu, S., Cinar, A., \& Lapsekili, N. (2013) Temperament and character properties of primary focal hyperhidrosis patients. Health and Quality of Life Outcomes, 11, 5. doi:10.1186/1477-7525-11-5

Ando', A., Pineda, J.A, Giromini, L., Soghoyan, G., Yang, Q., Bohm M., Maryanovsky, D., \& Zennaro, A. (2017). Effects of repetitive transcranial magnetic stimulation (rTMS) on attribution of movement to ambiguous stimuli and EEG mu suppression. Brain Research, 1680, 69-76. doi: 10.1016/j. brainres.2017.12.007

Ando', A., Salatino, A., Giromini, L., Ricci, R., Pignolo, C., Cristofanelli, S. ... Zennaro, A. (2015). Embodied simulation and ambiguous stimuli: The role of the mirror neuron system. Brain Research, 1629, 135-142. doi: 10.1016/j.brainres.2015. 10.025

Appenzeller, O. (1982). The autonomic nervous system. Amsterdam: Elsevier.

Bagby, R. M., Parker, J. D. A., \& Taylor, G. J. (1994). The twenty-item Toronto Alexithymia Scale-I. Item selection and cross-validation of the factor structure. Journal of Psychosomatic Research, 38, 23-32. doi:10.1016/00223999(94)90005-1

Birner, P., Heinzl, H., Schindl M., Pumprla, J., \& Schnider, P. (2000). Cardiac autonomic function in patients suffering from primary focal hyperhidrosis. European Neurology, 44, 112-116. doi: 10.1159/000008207

Bornstein, R. F. (2015). Personality assessment in the diagnostic manuals: On mindfulness, multiple methods, and test score discontinuities. Journal of Personality Assessment, 97, 446455. doi: 10.1080/00223891.2015.1027346

Bornstein, R.F. (2017). Evidence-Based Psychological Assessment. Journal of Personality Assessment, 99, 435-445. doi: 10.1080/00223891.2016.1236343.

Boucsein, W. (1992). Electrodermal activity, New York, NY: Plenum Press.

Boucsein, W. (2012). Electrodermal Activity (2nd ed.) New York, NY: Springer.

Bowlby, J. (1977). The making and breaking of affectional bonds: I. Aetiology and psychopathology in light of attachment theory. British Journal of Psychiatry, 130, 201-210.

Campbell, D. T., \& Fiske, D. V. (1959). Convergent and discriminant validation by the multitrait multimethod matrix. Psychological Bullettin, 56, 81-105. 
Cina, C. S., Robertson, S.G., Young, E.J., Cartier, B., \& Clase, C.M (2006). Effect of endoscopic sympathectomy for hyperhidrosis on quality of life using the illness intrusiveness rating scale. Minerva Chirurgica, 61, 231-239.

Cinà, C.S., \& Clase C.M (1999). The Illness Intrusiveness Rating Scale: a measure of severity in individuals with hyperhidrosis. Quality of Life Research, 8, 693-698.

Condren, R.M., O’Neill, A., Ryan, M.C.M., Barrett, P., \& Thakore J.H. (2002). HPA axis response to a psychological stressor in generalised social phobia. Psychoneuroendocrinology, 27, 693-703. doi: 10.1016/S0306-4530(01)00070-1.

De Marinis, M., Colaizzo, E., Petrelli, R. A. N., \& Santilli, V. (2012). Alterations in cardiovascular autonomic function tests in idiopathic hyperhidrosis. Autonomic Neuroscience, 167, 34-38.

Derogatis, L.R. (1994). Symptom Checklist-90-R: Administration, scoring, and procedures manual (3rd ed.). Minneapolis, MN: National Computer Systems.

Dickerson, S., \& Kemeny, M. (2004). Acute stressors and cortisol responses: a theoretical integration and synthesis of laboratory research. Psychological Bulletin, 130, 355-391. doi: 10.1037/0033-2909.130.3.355

Di Girolamo, M., Giromini L., Winters C. L., Serie C. M., \& de Ruiter, C. (2017). The Questionnaire of Cognitive and Affective Empathy: A Comparison Between Paper-and-Pencil Versus Online Formats in Italian Samples. Journal of Personality Assessment, 101(2), 159-170. doi: 10.1080/00223891. 2017.1389745.

Dozier, M., \& Kobak, R. R. (1992). Psychophysiology in attachment interviews: converging evidence for deactivating strategies. Annual Progress in Child Psychiatry and Child Development, 63 (6), 80-92.

Earle, T.L., Wolfgang, L., \& Weinberg, J. (1999). Differential effects of harassment on cardiovascular and salivary cortisol stress reactivity and recovery in women and men. Journal of Psychosomatic Research, 46, 125-141. doi: 10.1016/S0022-3999(98)00075-0.

Edwards, S., Lennox, G., Robson, K., \& Whiteley, A. (1996). Hypothermia due to hypothalamic involvement in multiple sclerosis. Journal of Neurology, Neurosurgery and Psychiatry, 61, 419-420.

Esen A. M., Barutcu, I., Karaca, S., Kaya, D., Kulac, M., Esen, O., ... Kilit, C. (2005). Peripheral vascular endothelial function in essential hyperhidrosis. Circulation Journal, 69, 707-710.

Fowles, D.C., Christie, M.J., \& Edelberg, R. (1981). Publication recommendations for electrodermal measurements. Psychophysiology, 18, 232-239.

Gander, M., \& Buchheim, A. (2015). Attachment classification, psychophysiology and frontal EEG asymmetry across the lifespan: a review. Frontiers in Human Neuroscience, 9, 116. doi: 10.3389/fnhum.2015.00079.

George, C., Kaplan, N., \& Main, M. (1996). Adult attachment interview. Unpublished manuscript. California: University of California at Berkeley.

Giromini, L., Ales, F., de Campora, G., Zennaro, A., \& Pignolo, C. (2017). Developing age and gender adjusted normative reference values for the difficulties in emotion regulation scale (DERS). Journal of Psychopathology and Behavioral Assessment. doi:/10.1007/s10862-017-9611-0

Giromini, L., Ando’ A., Morese, R., Salatino, A., Di Girolamo, M., Viglione D.J., \& Zennaro A. (2016a). Rorschach Performance Assessment System (R-PAS) and vulnerability to stress: A preliminary study on electrodermal activity during stress. Psychiatry Research, 246, 166-172. doi: 10.1016/j.psychres.2016.09.036.

Giromini, L., de Campora, G., Brusadelli, E., D’Onofrio, E., Zennaro, A., Zavattini G. C., \& Lang, M. (2016b). Validity and reliability of the Interpersonal Competence Questionnaire: Empirical evidence from an Italian study. Journal of Psychopathology and Behavioral Assessment, 38, 113-123. doi: 10.1007/s10862-015-9499-5

Giromini, L., Velotti, P., de Campora, G., Bonalume, L., \& Zavattini, G. C. (2012). Cultural adaptation of the Difficulties in Emotion Regulation Scale: Reliability and validity of an Italian version. Journal of Clinical Psychology 68, 9891007. doi: $10.1002 /$ jclp. 21876

Glogau, R. G. (2001). Treatment of palmar hyperhidrosis with botulinum toxin. Seminars in Cutaneous Medicine and Surgery, 20, 101-108. doi: 10.1053/sder.2001.25140.

Gratz, K. L., \& Roemer, L. (2004). Multidimensional assessment of emotion regulation and dysregulation: development, factor structure, and initial validation of the difficulties in emotion regulation scale. Journal of Psychopathology and Behavioral Assessment. doi: 10.1023/B:JOBA.0000007455.08539.94.

Gross, J. J. (1998). Antecedent- and response-focused emotion regulation: divergent consequences for experience, expression, and physiology. Journal of Personality and Social Psychology, 74, 224-237. doi: 10.1037/0022-3514.74.1.224

Gross, J. J., \& Levenson, R. W. (1997). Hiding feelings: The acute effects of inhibiting negative and positive emotion. Journal of Abnormal Psychology, 106, 95-103. doi: 10.1037/0021-843X.106.1.95

Haider, A., \& Solish, N. (2005). Focal hyperhidrosis: diagnosis and management. CMAJ. 17, 69-75. doi: 10.1503/ cmaj. 1040708

Healey, J. A., \& Picard, R.W. (2005). Detecting stress during real-world driving tasks using physiological sensors. IEEE Transactions on Intelligent Transportation System, 6, 156166. doi: 10.1109/TITS.2005.848368

Horowitz, L.M, Rosenberg, S.E., Baer, B.A., Ureño, G., \& Villaseñor, V.S. (1998). Inventory of interpersonal problems: psychometric properties and clinical applications. Journal of Consulting and Clinical Psychology, 56, 885-892. doi: 10.1037/0022-006X.56.6.885

Iwase, S., Ikeda, T., Kitazawa, H., Hakusui, S., Sugenoya, J., \& Mano, T. (1997) Altered response in cutaneous sympathetic outflow to mental and thermal stimuli in primary palmoplantar hyperhidrosis. Journal of the Autonomic Nervous System, 64, 65-73.

Ionescu-Tirgovişte, C., \& Prună, S. (1993). The pattern of the electrodermal activity as indicator of stress related reaction. Romanian Journal of Physiology, 30, 207-218.

Johnson, F. Y. A., \& Mostaghimi, H. (1995). Co-morbidity between dermatologic diseases and psychiatric disorders in Papua New Guinea. International Journal of Dermatology, 34, 244-248

Karaca, S., Emul, M., Kulac, M., Yuksel, S., Ozbulut, O., Guler, O., \& Gecici, O. (2007). Temperament and character profile in patients with essential hyperhidrosis. Dermatology, 214, 240-245. doi: 10.1159/000099589

Kaushik, R. M., Mahajan, S.K., Rajesh, V., \& Kaushik, R. (2004). Stress profile in essential hypertension. Hypertension Research, 27, 619-624. doi: 10.1291/hypres.27.619

Kirschbaum, C., Prüssner, J.C., Stone, A.A., Federenko, I., Gaab, J., Lintz, D., Schommer, N., \& Hellhammer, D.H. (1995). Persistent high cortisol responses to repeated psy- 
chological stress in a subpopulation of healthy men. Psychosomatic Medicine, 57, 468-474.

Lai, J., \& Linden, W. (1992). Gender, anger expression style, and opportunity for anger release determine cardiovascular reaction to and recovery from anger provocation. Psychosomatic Medicine, 54, 297-310. doi: 10.1097/00006842199205000-00006.

Lin, T.K., Chee, E. C.Y., Chen, H. J., \& Cheng, M. H. (1995) Abnormal sympathetic skin response in patients with palmar hyperhidrosis. Muscle Nerve, 18, 917-919 doi: 10.1002/ mus. 880180820

Mahendiran, S., Burkhart G.N., \& Burkhart, C. G. (2009). Hyperhidrosis: a review of a medical condition. The Open Dermatology Journal, 3, 195-197.

Manca, D., Valls-Sole J., Callejas M. A. (2000). Excitability recovery curve of the sympathetic skin response in healthy volunteers and patients with palmar hyperhidrosis. Clinical Neurophysiology, 111, 1767-1770. doi: 10.1016/S13882457(00)00394-1

McEwen, B. S. (1998). Stress, adaptation, and disease: Allostasis and allostatic load. Annals of the New York Academy of Sciences, 840, 33-44.

Meaney, M. J. (2001). Maternal care, gene expression, and the transmission of individual differences in stress reactivity across generations. Annual Review of Neuroscience, 24,1161-1192. doi: 10.1146/annurev.neuro.24.1.1161.

Mestanik, M., Visnovcova, Z., \& Tonhajzerova, I. (2014). The assessment of the autonomic response to acute stress using electrodermal activity. Acta Medica Martiniana, 14, 5-9. doi: 10.2478/acm-2014-0006.

Meyer, G. J. (2018). Paul Lerner Memorial Lecture: On Methods of Knowing in Psychology. [PowerPoint slides]. Society for Personality Assessment International Conference. Washington. Available from: https://www.youtube.com/watch?v=VkVAnDgg6yA\& $\mathrm{t}=228 \mathrm{~s}$

Meyer, G. J., Viglione, D. J., Mihura, J. L., Erard, R. E., \& Erdberg, P. (2011). A Manual for the Rorschach Performance Assessment System. Toledo, OH: R-PAS.

Mihura, J.L, Meyer, G.J., Dumitrascu, N., \& Bombel, G. (2013). The validity of individual rorschach variables: systematic reviews and meta-analyses of the comprehensive system. Psychological Bulletin, 139, 548-605. doi: 10.1037/a0029406

Naseem, Z. \& Khalid, R. (2010). Positive thinking in coping with stress and health outcomes: literature review. Journal of Research and Reflections in Education, 4, 46-61.

Papez, J. W. (1937). A proposed mechanism of emotion. Archives of Neurology and Psychiatry, 38, 725-43. doi: 10.1001/archneurpsyc.1937.02260220069003.

Pignolo, C., Giromini, L., Ando', A., Ghirardello, D., Di Girolamo, M., Ales, F., \& Zennaro, A. (2017). An interrater reliability study of rorschach performance assessment system (R-PAS) raw and complexity-adjusted scores. Journal of Personality Assessment, 1-7. doi: 10.1080/00223891.2017. 1296844

Reniers, R. L. E. P., Corcoran, R., Drake, R., Shryane, N. M., \& Völlm, B. A. (2011). The QCAE: A Questionnaire of Cognitive and Affective Empathy. Journal of Personality Assessment, 93, 84-95, doi: 10.1080/00223891.2010.528484

Ricchetti-Masterson, K., Symons, J. M., Aldridge, M., Patel, A., Maskell, J., Logie, J., Yamaguchi, Y., \& Cook, S. F. (2018). Epidemiology of hyperhidrosis in 2 population-based health care databases. Journal of the American Academy of Dermatology, 78, 358-362. doi: 10.1016/j.jaad.2017.10.004.
Roisman, G. I. (2007). The psychophysiology of adult attachment relationships: autonomic reactivity in marital and premarital interactions. Developmental Psychology, 43, 39-53. doi: 10.1037/0012-1649.43.1.39

Ruchinskas, R. A., Narayan, R. K., Meagher, R. J., \& Furukawa, S. (2002). The relationship of psychopathology and hyperhidrosis. British Journal of Dermatology, 147, 733-735.

Saarni, A. (1999). The relationship between type A personality and experienced stress with heart attack. Psychological Research, 4, 3-14.

Sayeed, R. A., Nyamekye, I., Ghauri, A. S., \& Poskitt, K. R. (1998). Quality of life after transthoracic endoscopic sympathectomy for upper limb hyperhidrosis. European Journal of Surgery Supplement, 164, 39-42.

Schmidt, S., Walach, H. (2000). Electrodermal activity (EDA): state-of-the-art measurement and techniques for parapsychological purposes. Journal of Parapsychology, 64, 139-163.

Shapiro, D. (1965). Stili Nevrotici. [Tr. it] Roma: Astrolabio.

Shapiro, D. (1981). Autonomy and rigid character. New York: Basic Books.

Shapiro, D. (1989). La personalità nevrotica. [Tr. it.] Torino: Bollati Boringhieri.

Shapiro, D. (2002). Dynamics of character: Selfregulation in psychopathology. New York: Basic Books.

Solish, N., Benohanian, A., \& Kowalski, J.W. (2005). Canadian Dermatology Study Group on health-related quality of life in primary axillary hyperhidrosis. Dermatology Surgery, 4, 405-413.

Spielberger, C. D., Gorsuch, R. L., Lushene, R. E., Vagg, P. R., \& Jacobs, G. A. (1983). Manual for the State-Trait Anxiety Inventory STAI (Form Y). Palo Alto: Consulting Psychologists Press.

Strutton, D.R., Kowalski, J.W., Glaser, D.A., \& Strang, P.E. (2004) US prevalence of hyperhidrosis and impact on individuals with axillary hyperhidrosis: Results from a national survey. Journal of the American Academy of Dermatology, 51, 241-248. doi: 10.1016/j.jaad.2003.12.040

Taylor, G. J., Bagby, R. M., \& Parker, J. D. A. (1997). Disorders of affect regulation: Alexithymia in medical and psychiatric illness. Cambridge: Cambridge University Press.

Trapnell, P. D., \& Campbell, J. D. (1999). Private self-consciousness and the fivefactor model of personality: Distinguishing rumination from reflection. Journal of Personality and Social Psychology, 76(2), 284-304.

Tronstad, C., Gjein, G. E., Grimnes, S., Martinsen, Ø. G., Krogstad, A. L., \& Fosse, E. (2008). Electrical measurement of sweat activity. Physiological Measurement, 29(6), S407.

Vetrugno, R., Liguori, R., Cortelli, P., \& Montagna, P. (2003). Sympathetic skin response: basic mechanisms and clinical applications. Clinical Autonomic Research, 13, 256-270. doi: 10.1007/s10286-003-0107-5

Vorkamp, T., Foo F. J, Khan, S., Schmitto, J. D., \& Wilson P. (2010). Hyperhidrosis: evolving concepts and a comprehensive review. Surgeon, 8, 287-282. doi: 10.1016/j.surge. 2010.06.002

Wigley, F.M. \& Flavahan, N.A. (2016). Raynaud's Phenomenon. The New England Journal of Medicine, 11, 556-565. doi: 10.1056/NEJMra1507638.

Won, K., Im, K., Ho, C., Won, K., Hin, S., Oun, Y., \& Hung, C. (1998). Essential hyperhidrosis: no evidence of abnormal personality features. Clinical and Experimental Dermatology, 23(1), 45-46. 